\title{
Eigenvalue distributions in Yang-Mills integrals
}

\author{
Werner Krauth ${ }^{\text {a.1 }}$, Matthias Staudacher ${ }^{6.2}$ \\ "CNRS-Laboratoire de Physique Statistique. Ecole Normale Supérieure, 24, rue Lhomond. F-75231 Paris Cedex 05. France \\ ${ }^{\mathrm{b}}$ Albert-Einstein-Institut, Max-Planck-Institut für Gracitationsphysik, Schlaatzweg 1, D-14473 Potsdam, Germany
}

Received 8 March 1999

Editor: L. Alvarez-Gaumé

\begin{abstract}
We investigate one-matrix correlation functions for finite $S U(N)$ Yang-Mills integrals with and without supersymmetry. We propose novel convergence conditions for these correlators which we determine from the one-loop perturbative effective action. These conditions are found to agree with non-perturbative Monte Carlo calculations for various gauge groups and dimensions. Our results yield important insights into the eigenvalue distributions $\rho(\lambda)$ of these random matrix models. For the bosonic models, we find that the spectral densities $\rho(\lambda)$ possess moments of all orders as $N \rightarrow \infty$. In the supersymmetric case, $\rho(\lambda)$ is a wide distribution with an $N-$ independent asymptotic behavior $\rho(\lambda) \sim \lambda^{-3}, \lambda^{-7}, \lambda^{-15}$ for dimensions $D=4,6,10$, respectively. (C) 1999 Published by Elsevier Science B.V. All rights reserved.
\end{abstract}

Recently there has been renewed interest in dimensional reductions of $S U(N)$ Yang-Mills theories. It has been argued that these "toy models", apart from some subtleties, allow to recover the full unreduced theories in the large $N$ limit [1]. The supersymmetric reductions are relevant to D-brane physics and have also been used in various attempts to define non-perturbative formulations of quantum gravity, supermembranes and superstrings. For the complete reduction, all space-time dependence is eliminated from the gauge "fields", and the continuum Yang-Mills path integral becomes an ordinary

\footnotetext{
'E-mail: krauth@physique.ens.fr

${ }^{2}$ E-mail: matthias@aei-potsdam.mpg.de; Supported in part by EU Contract FMRX-CT96-0012.
}

multi-matrix integral. The integrals appear to be ill-defined due to the flat directions (i.e. commuting matrices) in the action. Recent calculations have however uncovered that in many cases of interest these matrix integrals converge and thus do not need to be regulated. This was analytically found in the supersymmetric case for $S U(2)$ in [2]. In $[3,4]$ we found by Monte Carlo methods that the absolute convergence persists for larger values of $N$. Furthermore, we numerically established (and proved analytically for $S U(2)$ ) the convergence properties of the bosonic (non-supersymmetric) integrals. Perturbative one-loop estimates in favor of convergence were presented for the supersymmetric case in [5] and, recently, in [6], for the bosonic case. Mathematically rigorous proofs for $N>2$ are however still missing. For some applications of these integrals see e.g. [2,7-9]. 
The integrals in question are

$$
\begin{aligned}
\mathscr{Z}_{D . N}^{\prime{ }^{\prime}}:= & \int \prod_{A=1}^{N^{2}-1}\left(\prod_{\mu=1}^{D} \frac{d X_{\mu}^{A}}{\sqrt{2 \pi}}\right)\left(\prod_{\alpha=1}^{W_{\alpha}} d \Psi_{\alpha}^{A}\right) \\
& \times \exp \left[\frac{1}{2} \operatorname{Tr}\left[X_{\mu}, X_{\nu}\right]\left[X_{\mu}, X_{\nu}\right]\right. \\
& \left.+\operatorname{Tr} \Psi_{\alpha}\left[\Gamma_{\alpha \beta}^{\mu} X_{\mu}, \Psi_{\beta}\right]\right] .
\end{aligned}
$$

They correspond to fully reduced $D$-dimensional Euclidean $S U(N)$ Yang-Mills theory. Here $\mathscr{N}$ is the number of real supersymmetries, and for $\mathscr{N}>0$ the only possible dimensions are $D=3,4,6,10$ corresponding to $\mathscr{N}=2,4,8,16$, respectively. If $\mathscr{N}=0$ (no supersymmetry), a priori all $D \geq 2$ are possible and we simply omit the terms with Grassmann fields both from the measure and action of Eq. (1). For the detailed notation we refer to $[3,4]$.

In view of [2-4] the necessary and sufficient conditions of existence for the integrals Eq. (1) then appear to be

$$
\left.\begin{array}{lll}
D=4,6,10 & \text { and } & N \geq 2 \\
D=3 & \text { and } & N \geq 4 \\
D-4 & \text { and } & N \geq 3 \\
D \geq 5 & \text { and } & N \geq 2
\end{array}\right\} \text { for } \mathscr{N}=0 .
$$

As already mentioned in [4], it is only the bosonic $D=3 S U(3)$ integral which escapes a clear-cut classification by numerical means as we cannot exclude almost marginal convergence. We have now checked that the susy $D=3$ integral is not absolutely convergent ${ }^{3}$ for any $N$, disproving a conjecture made in [4].

For the large $N$ limit, Eq. (2) implies existence of the integrals for $D \geq 3$ in the bosonic case and for $D=4,6,10$ in the supersymmetric case.

Analytic motivation of Eq. (2) for general $N$ can be obtained from the one-loop perturbative calcula-

\footnotetext{
${ }^{3}$ This case, with $\mathscr{N}=2$, is special in that, at least for even $N$, the integral is formally zero[3]. The integral is however not absolutely convergent, as we have checked numerically with the methods used in this paper. This divergence is also present in the analytically tractable $S U(2)$ case. Adding a Chern-Simons term, as proposed in [10]. does not improve convergence and the $D=3$ susy integral modified by such a term is divergent for all $N$.
}

tions of the effective action as presented in $[5,6]$ : Decompose the matrices into diagonal $D\left(X_{\mu}\right)$ (with matrix elements $\left.D\left(X_{\mu}\right)_{i j}=\delta_{i,} x_{\mu}^{i}\right)$ and off-diagonal components $Y_{\mu}$ (i.e. $\left.\left(Y_{\mu}\right)_{i i}=0\right): X_{\mu}=D\left(X_{\mu}\right)+Y_{\mu}$. Fix a background gauge, expand the action to quadratic order in $Y_{\mu}$ and work out the cffective action for the diagonal elements $x_{\mu}^{i}$. The result for the supersymmetric case [5] reads

$$
\begin{aligned}
\mathscr{Z}_{D, N}^{v} \sim & \int \prod_{i, \mu}^{N, D} d x_{\mu}^{i}\left[\prod_{\mu} \delta\left(\sum_{i} x_{\mu}^{i}\right)\right] \\
& \times \sum_{G: \text { maximal tree }(i j): \text { link of } G} \frac{1}{\left(x^{i}-x^{j}\right)^{3(D-2)}} \\
& +\ldots
\end{aligned}
$$

Here the sum is over all possible maximal (i.e. there are $N-1$ links in $G$ ) trees $G$ connecting the $N$ coordinates. As indicated in Eq. (3), there are actually further terms present to one-loop order for $D=$ 6,10 , but they are irrelevant for the powercounting arguments below (see [5]). For the bosonic case [6] one finds

$$
\begin{aligned}
\mathscr{Z}_{D, N}^{(-1)} \sim & \int \prod_{i, \mu}^{N . D} d x_{\mu}^{i}\left[\prod_{\mu} \delta\left(\sum_{i} x_{\mu}^{i}\right)\right] \\
& \times \prod_{i<j} \frac{1}{\left(x^{i}-x^{j}\right)^{2(D-2)}}
\end{aligned}
$$

The one-loop approximation is reasonable for wellseparated diagonal components $x_{\mu}^{i}$ (that is the "infrared" regime). It is now easily verified that superficial powercounting of all integrals on hyperplanes of the integration space in Eq. (3), Eq. (4) results, at large $x_{\mu}^{i}$, precisely in the convergence conditions Eq. (2).

Clearly, this perturbative argument is not a proof of Eq. (2): First, the cumulative effect of all corrections to this one-loop effective action is beyond control. Second, there are potentially dangerous regions in the integration space where some of the differences $\left(x^{i}-x^{j}\right)^{2}$ are small (thus rendering the one-loop approximation invalid) while others are large (leading to a potential divergence).

An analytic calculation of the $D=4,6,10$ susy partition functions $\mathcal{Z}_{\hat{N}, D}^{r}$ has been reported in [10]. 
In this work, light-cone variables $\phi=X_{1}+i X_{D}, \bar{\phi}$ $=X_{1}-i X_{D}$ are used. Subsequently, $\phi$ and $\bar{\phi}$ are treated as independent hermitian matrices (i.e. one actually has $\phi=X_{1}-X_{0}, \bar{\phi}=X_{1}+X_{0}$ ), and the integrals computed in [10] correspond to Eq. (1) with Minkowski (as opposed to Euclidean) metric. However, the Minkowski integrals are divergent and need to be regulated. This calculation might be rendered rigorous if one succeeded in deriving the imposed pole prescriptions directly from the matrix Wick rotation $X_{D} \rightarrow i X_{0}$.

For applications, it is important to understand the statistical eigenvalue distribution of these random matrix models. The simplest quantity is the distribution for the eigenvalues $\lambda_{i}$ of just one matrix, say, $X_{1}$. in the background of the other matrices $X_{2}, \ldots, X_{D}$

$\rho(\lambda)=\frac{1}{N}\left\langle\sum_{i=1}^{N} \delta\left(\lambda-\lambda_{i}\right)\right\rangle$.

Here, the average $\langle>$ is with respect to Eq. (1). The easiest way to investigate the density is to consider the one-matrix correlators ${ }^{+}$

$$
\left\langle\frac{1}{N} \operatorname{Tr} X_{1}^{2 k}\right\rangle=\int_{-\infty}^{\infty} d \lambda \rho(\lambda) \lambda^{2 k} .
$$

In an ordinary hermitian matrix model, say with weight $\exp \left(-\operatorname{Tr} X_{1}^{2}\right)$, the density Eq. (5) falls off at infinity as $\rho(\lambda) \sim \exp \left(-\lambda^{2}\right)$. Therefore all moments Eq. (6) exist.

In the Yang-Mills ensembles for $S U(2)$, we obtained analytically (along the lines of [2]) that the correlators Eq. (6) exist only for low values of $k$; we find $k<D-3(\mathscr{N}>0)$ and $k<\frac{D}{2}-2(\mathscr{N}=0)$. For general $N$, we obtain the convergence conditions in the one-loop approximations Eq. (3), Eq. (4). Some care has to be exercised, since the most divergent contribution is not obtained by simply counting the overall dimensionality of the integral. In fact, a more dangerous infrared configuration stems from

\footnotetext{
${ }^{4}$ One might attempt to compute such correlators within the powerful framework of [10]. The natural candidate would be $\left\langle\frac{1}{N} \operatorname{Tr} \phi^{k}\right\rangle$ (where $\phi=X_{1}+i X_{D}$ ). Unfortunately, these correlators vanish for all $k$ in the Euclidean integral Eq. (1) due to the $S O(2)$ symmetry in the $\left(X_{1}, X_{D}\right)$ plane [11].
}

only a single (say, the $i$-th) eigenvalue $x_{\mu}^{i}$ becoming large in the $D$-dimensional space. The only exception being, once again, the bosonic $D=3 \quad S U(3)$ integral. Therefore, apart from this one case, we find

$$
\begin{aligned}
& \left\langle\frac{1}{N} \operatorname{Tr} X_{1}^{2 k}\right\rangle<\infty \\
& \operatorname{iff}\left\{\begin{array}{ccc}
k<D-3 & \text { for } & \mathscr{N}>0, \\
k<N(D-2)-\frac{3}{2} D+2 & \text { for } & \mathscr{N}=0 .
\end{array}\right.
\end{aligned}
$$

This means that no moments exist for the $D=4$ susy integral, and only the first two and six even moments for, respectively, the $D=6$ and $D=10$ susy integrals!

We have been able to directly verify our conjecture Eq. (7) with the Monte Carlo approach of $[3,4]$. It may seem straightforward to obtain a direct numerical estimate of the eigenvalue spectrum of $X_{1}$ by generating ensembles of $X=\left(X_{1}, X_{2}, \ldots, X_{D}\right)$ with the statistical weight

$$
z_{D, N}^{\nu}(X)=\exp \left[\frac{1}{2} \operatorname{Tr}\left[X_{\mu}, X_{\nu}\right]\left[X_{\mu}, X_{\nu}\right]\right] \mathscr{P}_{D, N}(X)
$$

where $\mathscr{P}_{D, N}(X)$ is the Pfaffian polynomial coming from the integration over the fermionic degrees of freedom (cf. [3], for the bosonic integrals, this term is simply dropped). The eigenvalue spectrum of $X$ can in principle be sampled and its histogram generated. In practice, it is however impossible to make firm predictions on the tails of the histogram, as they comprise an exceedingly small number of samples. For this reason, we rather generate Markov chains of configurations $X(t)$ with the statistical weight

$\pi_{D, \dot{N}}^{\cdot k}(X)=\left|\sum_{\mu} \operatorname{Tr} X_{\mu}^{2 k} z_{D, N}(X)\right|$

This means in particular that we perform one independent run for every value of $k$. The critical information on the convergence condition is contained in the autocorrelation function of our Markov-chain vector $X(t)$

$f_{t}^{\Delta}-\frac{X(t) \cdot X(t-\Delta)}{\sqrt{X(t-\Delta) \cdot X(t-\Delta)} \sqrt{X(t) \cdot X(t)}}$ 

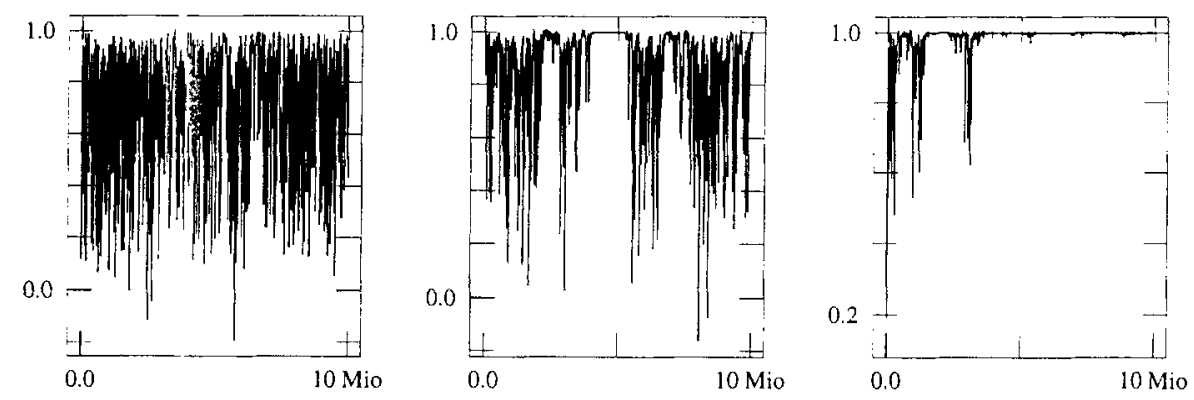

Fig. 1. Autocorrelation functions $f_{t}^{3}$ versus Monte Carlo time $t(\Delta=1000)$ for the bosonic integral with $N=6, D=3$, and, from the left, $k=2,3,4$.

for a fixed value of $\Delta$ (as in [4], we have compactified all the integrands). An absolutely non-integrable singularity of the integrand corresponds to an infinite statistical weight concentrated in a region of negligible extension. This simply means that the simulation should get stuck, and the autocorrelation function approach 1. An integrable singularity is not picked up by the present method.

We have computed the autocorrelation function for a large number of cases. Among the supersymmetric cases, we considered $N \leq 6$ for $D=4, N \leq 5$ for $D=6$ and $N=2,3,4$ for $D=10$. All the analytically known results for $S U(2)$ were easily reproduced. Convergence of bosonic integrals was checked for $N \leq 9$ and $D=3,4,6$.

As an example, we show in Fig. 1 the autocorrelation functions of typical runs for the bosonic integrals with $D=3, N=6$ for $k=2, k=3$ and $k=4$. The criterion Eq. (7) leads us to expect convergence for $k=2$ and $k=3$, as the smallest diverging power is $k_{s, r i s}=3.5$. This behavior is clearly reproduced in Fig. 1. Notice that the "almost" divergent case $k=3$ gets stuck for very long periods of Monte-Carlo time, whereas the the almost "convergent" integral $k=4$ settles to a unit value of $f_{t}^{\delta}$ only after a very long transient.

In Fig. 2, we show corresponding plots for the supersymmetric case with $D=6, N=4$. Here, we expect the $k=2$ integral to be convergent, as clearly found, while $k=4$ is divergent, as expected. In the marginally divergent case, we have observed the typical alternations between "stuck" and "mixing" behavior.

Notice that the middle plots of Fig. 1 and Fig. 2 do not appear fundamentally different, even though we expect the integrals to behave differently. Our Monte Carlo procedure leaves a margin of error which we estimate to be $\Delta k \sim \pm 1 / 2$. A similar
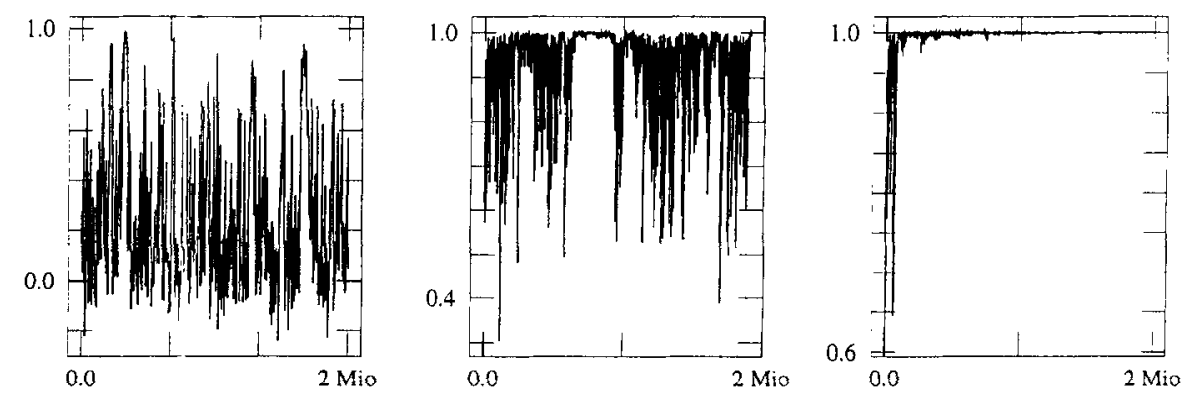

Fig. 2. Autocorrelation functions $f_{1}^{\Delta}$ versus Monte Carlo time $t(A=100)$ for the supersymmetric integral with $D=6, N=4$, and, from the left, $k=2,3,4$. 
ambiguity affects the abovementioned bosonic integral for $D=3$ and $N=3$.

The conditions Eq. (7) mean that in the YangMills case the asymptotic behavior of the one-matrix eigenvalue distribution Eq. (5) decays algebraically:

$\rho(\lambda) \sim|\lambda|^{-\alpha}$ for $\lambda \rightarrow \pm \infty$.

Assuming this to be exactly true we can extract the power $\alpha$ in Eq. (11):

$\alpha=\left\{\begin{array}{cc}2 D-5 & \text { for } \quad \mathscr{N}>0, \\ 2 N(D-2)-3 D+5 & \text { for } \quad \mathscr{N}=0 .\end{array}\right.$

Most strikingly, the decay of the densities in the susy cases $D=4.6,10\left(\rho(\lambda) \sim \lambda^{-3}, \lambda^{-7}, \lambda^{-15}\right)$ is independent of $N$. It means that the eigenvalue distribution are wide even in the $N \rightarrow \infty$ limit! This is a most unusual effect for a random matrix model. Evidently, supersymmetry is responsible for this behavior, as can e.g. be seen by comparing the one-loop effective actions for the diagonal matrix elements in the susy (cf. Eq. (3)) and non-susy (cf. Eq. (4)) case: In the latter, this effective action is $\mathscr{O}\left(N^{2}\right)$ while in the former it is $\mathscr{O}(N)$.

The bosonic case is much more conventional in that the density becomes concentrated in a finite interval at large $N$. In this context it is interesting to mention the result of a numerical study of the bosonic Yang-Mills integrals which is complementary to the present work: In [6], Hotta et al. investigate the scaling behavior of $\left\langle\frac{1}{N} \operatorname{Tr} X_{1}^{2}\right\rangle$ (i.e. Eq. (6) with $k=1)$ with $N$. They find that, if the variables in Eq. (1) are rescaled as $X_{\mu}^{A} \rightarrow N^{\frac{1}{4}} X_{\mu}^{A}$ such that an explicit factor of $N$ appears in front of the action, this correlator tends to a constant as $N \rightarrow \infty$. In consequence, the usual 't Hooft scaling of large $N$ matrix models is obeyed, and the edge of the eigenvalue support tends to a constant. Nevertheless, the observed asymptotic behavior $\left(\rho(\lambda) \sim \lambda^{-2 N(D-2)}\right.$ as $N \rightarrow \infty$ ) is different from the universal exponential decay law of Wigner-type random systems.

To summarize, we have demonstrated how statistical information on the eigenvalue distributions of Yang-Mills integrals (which are the simplest exam- ples for the so-called "new" matrix models) can be obtained. We observed an interesting difference in the tails of the spectral distribution between the susy and non-susy ensembles. While suggestive - e.g. we find it interesting that the susy eigenvalue distribution tends to stretch out much farther - the present study clearly does not yet address such important issues as the full nonperturbative effective action for the diagonal elements of all $D$ matrices or the problem of level spacing statistics. These questions will need to be addressed if one intends to apply Yang-Mills integrals to string theory and large $N$ gauge theory.

\section{Acknowledgements}

We thank J. Hoppe, V. A. Kazakov, I. K. Kostov, H. Nicolai and J. Plefka for useful discussions. M. S. thanks the LPS-ENS Paris for hospitality. This work was supported in part by the EU under Contract FMRX-CT96-0012.

\section{References}

[1] T. Eguchi, H. Kawai, Phys. Rev. Lett. 48 (1982) 1063.

[2] P. Yi. Nucl. Phys. B505 (1997) 307, hep-th/9704098; S. Sethi. M. Stern. D-Brane Bound State Redux. hepth/ 9705046 .

[3] W. Krauth, H. Nicolai, M. Staudacher. Phys. Lett. B431 (1998) 31, hep-th /9803117.

[4] W. Krauth, M. Staudacher. Phys. Lett. B435 (1998) 350. hep-th $/ 9804199$.

[5] H. Aoki, S. Iso, H. Kawai, Y. Kitazawa, T. Tada, Prog. Theor. Phys. 99 (1998) 713, hep-th/9802085.

[6] T. Hotta, J. Nishimura, A. Tsuchiya, Dynamical Aspects of Large $N$ Reduced Models, hep-th/9811220.

[7] N. Ishibashi, H. Kawai, Y. Kitazawa, A. Tsuchiya. Nucl. Phys. B498 (1997) 467, hep-th/9612115.

[8] I.K. Kostov, P. Vanhove, Phys. Lett. B444 (1998) 196. hep-th $/ 9809130$.

[9] N. Dorey, T.J. Hollowood, V.V. Khoze. M.P. Mattis, S. Vandoren, Multi-Instantons and Maldacena's Conjecture. hep-th/9810243: Multi-Instanton Calculus and the AdS /CFT Correspondence in $\mathscr{N}=4$ Superconformal Field Theory, hep-th/9901128.

[10] G. Moore, N. Nekrasov, S. Shatashvili, D-particle bound states and generalized instantons, hep-th/9803265.

[11] M. Douglas, private communication. 